\title{
The Role of HPV Oncogenes in the Regulation of microRNA pathway in Cervical Cancer
}

\author{
Yi Song, Tao Tang, Yan Deng, Yi Zhao \\ Obstetrics \& Gynaecology, The Chinese University of Hong Kong, China
}

High risk Human Papillomavirus (HPVs) infection is the etiological agent in cervical cancer. HPV types have been subdivided into low-risk types, which are found mainly in genital warts, and high-risk types, which are frequently associated with invasive cervical cancer. HPV16 and HPV18 count for 70\% of cervical cancer. The E6, E7 genes in the early region of HPV genome are proved to have a significant impact on progression of cervical cancer. miRNA is a kind of short (about 22nt) non-coding RNA which regulates the gene expression on post-transcriptional and translational level, and it has been proved to have a significant impact on tumor development. Our group previously investigated the correlation of miRNAs and HPV oncogenes, and identified several differentially expressed miRNAs in cervical cancer cell lines (HeLa, SiHa, C33A, ME180, Caski, CC2 and CC3) as well as clinical specimens, including 2 up-regulated and 9 down-regulated. We further investigate the direct relationship between the oncogenes and miRNAs. C33A cells were transfected with single E6/E7 vector or E6/E7 vector + E6/E7 siRNA, and the expression of miRNAs were tested through qRT-PCR. The result of the relative expression level of miRNAs shows some consistency. The HPV oncogenes could down-regulate the expression of miR-145, miR-195 and miR-142-3p. We also have examined the oncogenetic effects of miR-145, miR-195 and miR-142-3p by MTT assay, which shows they all reduce the cell viability. Further investigation will be done for the mechanisms. The work could provide new insight in diagnosis and treatment for cervical cancer. 